\title{
Mulibrey nanism: clinical features and diagnostic criteria
}

\author{
N Karlberg, H Jalanko, J Perheentupa, M Lipsanen-Nyman
}

J Med Genet 2004;41:92-98. doi: 10.1136/jmg.2003.014118

Mulibrey nanism (MUL) is an autosomal recessive disease caused by mutations in the TRIM37 gene encoding the peroxisomal TRIM37 protein of unknown function. In this work, we analysed the clinical characteristics of 85 Finnish patients with MUL, most of whom were homozygous for the Finn major mutation of TRIM37. The patients' hospital records from birth to the time of the diagnosis at age 0.0252 years (median 2.1 years) were retrospectively analysed. All except four of the patients (95\%) had a prenatal onset growth failure without postnatal catch up growth. The mean length standard deviation score (SDS) was -3.1 and -4.0 at birth and at diagnosis, respectively. In infancy, feeding difficulties, and respiratory tract infections were the most common problems. Congestive heart failure and pericardial constriction were diagnosed during infancy in $12 \%$ and $6 \%$ of the patients, respectively. At the time of the diagnosis, characteristic craniofacial features of scaphocephaly, facial triangularity, high and broad forehead, and low nasal bridge were evident in over $90 \%$ of the patients. In addition, practically all patients were gracile and had thin extremities. Other findings included a peculiar high-pitched voice (96\%), yellowish dots in ocular fundi $(79 \%)$, cutaneous naevi flammei $(65 \%)$, hepatomegaly $(45 \%)$, and fibrous dysplasia of long bones (25\%). Mild muscular hypotonicity (68\%) was the only neurological abnormality. The clinical features of the Finnish patients with MUL formed a distinct entity. The most consistent findings were growth failure and characteristic craniofacial features. However, organ manifestations varied considerably in early childhood. Based on these findings, we propose new diagnostic criteria for MUL.

See end of article for authors' affiliations

Correspondence to: Correspondence to:
Dr M Lipsanen-Nyman The Hospital for Children and Adolescents, University of Helsinki, 00029 HYKS, Finland: marita.lipsanen@hus.fi
M ulibrey nanism (muscle-liver-brain-eye nanism, MUL; OMIM \#253250) is an autosomal recessive disorder with severe growth failure and multiple organ manifestations first described in the early 1970s. ${ }^{12}$ Today we know of some 110 patients, 85 of them from Finland. Sporadic cases have been reported from various ethnic groups all over the world. ${ }^{3-12}$

MUL is caused by mutations in the TRIM37 gene on chromosome $17 \mathrm{q} 22-\mathrm{q} 23 .{ }^{13}$ Five different mutations have been reported in patients with MUL. Two of them are Finnish, the Finn major (c.493-2A $\rightarrow \mathrm{G}$ ) and the Finn minor mutation (c.2212delG). The others are Czech (c.838842delACTTT), American (c.1346-1347insA) ${ }^{13}$ and Turkish (c.855_862delTGAATTAG)..$^{22}$ All five mutations result in a shift of the reading frame, resulting in a truncated TRIM37 protein. ${ }^{13}$ TRIM37 is a $130 \mathrm{kDa}$ protein expressed in many tissues $^{14}$ and located in the peroxisomes, suggesting that MUL is a peroxisomal disease..$^{15}$ TRIM37 belongs to a new subfamily of zinc finger proteins (previously designated RBCC for ring-B box-coiled coil). The function of the TRIM37 protein and the pathogenetic mechanisms underlying MUL are unknown.

Diagnostic evaluation and clinical care of the patients with MUL in Finland has been centred in our institution. Here we report the clinical findings from birth to the time of the diagnosis in all known Finnish patients, and present revised diagnostic criteria.

\section{PATIENTS AND METHODS \\ Patients}

The series included all the known 85 Finnish patients with MUL diagnosed by the original criteria. ${ }^{2}$ DNA was available from 80 patients; 78 of them homozygous for the Finn major mutation, and two compound heterozygotes for the two Finnish mutations. The other five patients had died before mutation analysis became available. In four of those five cases, DNA was obtained from family member(s); one parent couple, two single parents, and one sibling, all carriers of the Finn major mutation. The phenotype of the remaining patient left no doubt about the diagnosis.

\section{Collection of data}

The hospital ethics committee approved the study. We retrospectively analysed the patients' hospital records from birth to the time of diagnosis. We recorded data on their clinical events and on their appearance and dysmorphic features. The patients were photographed, and $x$ rays of the skull, thorax, and long bones as well as appropriate laboratory assays were obtained on the first visits. The obstetric and growth histories were collected from hospitals and child welfare centres. The patients' parents, relatives, and physicians were interviewed for missing details. Birth size and subsequent lengths/ heights were expressed as standard deviation scores (SDS) according to the Finnish standards, and postnatal weights as percentage deviations from the mean weight for height and sex.

Abbreviations: SDS, standard deviation score; SGA, small for gestational age; SRS, Silver-Russell syndrome 


\section{RESULTS}

The clinical data on the 85 Finnish patients with MUL were systematically analysed from birth to time of the diagnosis. The age at the diagnosis varied from 0.02 to 52 years (median 2.1 years). A third of them were younger than 1 year and two thirds were younger than 5 years.

\section{Pregnancy and delivery}

Most of the pregnancies were uneventful. Complications included pre-eclampsia (three cases), anti-Rhesus immunisation (a pair of siblings), gestational diabetes (one), and varicella zoster infection (one). Nineteen of the pregnancies (23\%) had been monitored because of poor fetal growth. Fifteen mothers $(18 \%)$ had had miscarriages in previous pregnancies. Length of gestation varied from 32 to 42 weeks (median 39 weeks); $90 \%$ of the children were born at full term. Labour was uncomplicated in all but three cases, in which an urgent caesarean section was performed because of threatened asphyxia. Apgar score at 5 minutes was on average 8 (range $2-10$ ).

\section{Newborn period}

Of the newborns, 81 (95\%) were small for gestational age (SGA), with birth length SDS below -2.0 for gestational age (fig 1). Birth length SDS was on average -3.1 (range -6.4 to 0.7 ), and mean birth weight SDS was -2.8 (range -4.0 to $0.5)$. Occipitofrontal head circumference SDS ranged from -0.9 to 0.8 (mean -0.5 ) indicating macrocephaly relative to length in the majority of children.

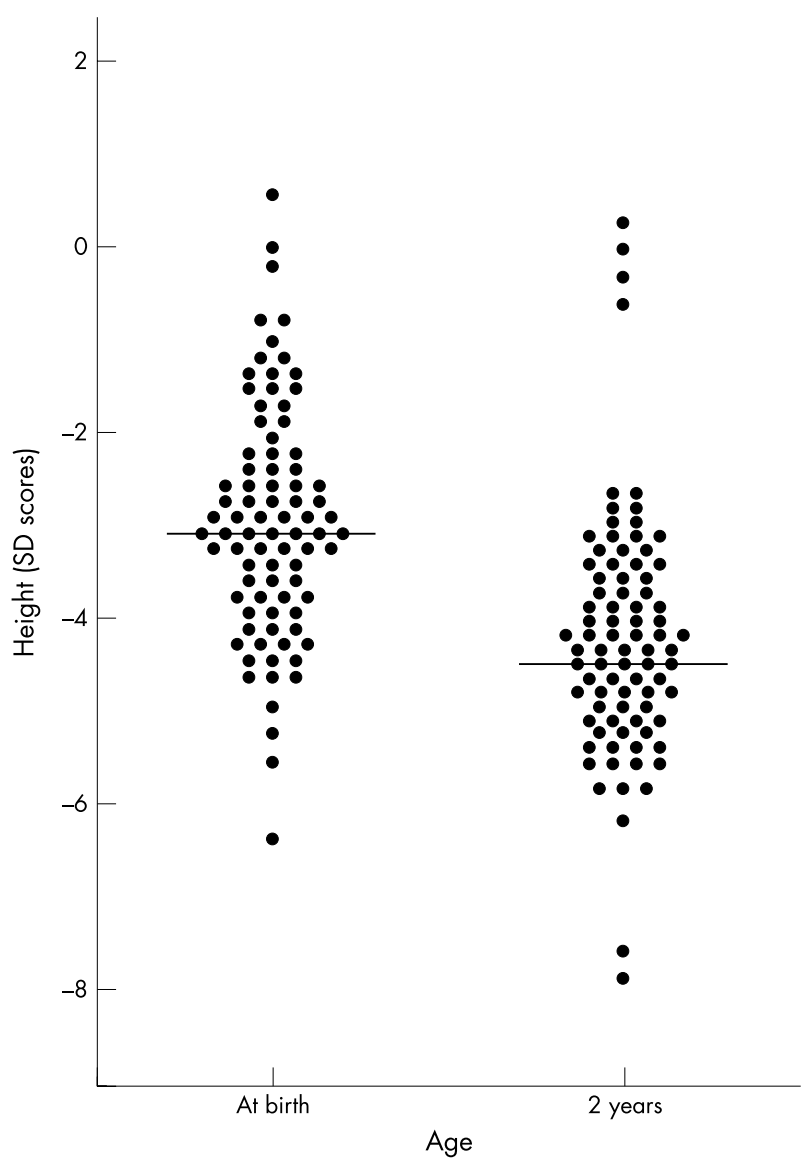

Figure 1 Length SDS at birth and at the age of 2.0 years in 85 infants with MUL, 39 boys and 46 girls. The growth failure progressed postnatally in $74 \%$ of the patients.

\begin{tabular}{ll} 
Table 1 Problems in infancy in 85 patients \\
with Mulibrey nanism \\
\hline Feature & Frequency (\%) \\
\hline Neonatal problems & \\
Respiratory problems & 31 \\
Ventilatory assistance needed & 20 \\
Cyanosis while crying & 15 \\
Feeding difficulties & 30 \\
Nasogastric feeding tube needed & 17 \\
Suspicion of hydrocephalus & 6 \\
Suspicion of sepsis & 6 \\
Cardiac arrhythmia & 1 \\
Growth failure & 96 \\
Infections & \\
Upper respiratory infections $>4 /$ year & 51 \\
Pneumonia & 47 \\
Necessitating hospital care & 30 \\
Three or more pneumonias & 27 \\
Ventilatory assistance needed & 25 \\
Intubation needed & 14 \\
Middle ear infections $>4 /$ year & 38 \\
Feeding difficulties & 50 \\
Vomiting & 39 \\
Difficulties in sucking & 31 \\
Nasogastric feeding tube needed & 31 \\
Delay in switching to solid foods & 24 \\
Fatigue during eating & 21 \\
Percutaneous gastrostomy needed & 6 \\
Muscular hypotonicity & 46 \\
Suspicion of hydrocephalus & 16 \\
Hypoglycaemia & 15 \\
Congestive heart failure & 11 \\
Resuscitation during first year of life & 11 \\
Due to infection & 9 \\
Due to aspiration & 1 \\
Due to arrhythmia & 1 \\
Death during infancy & 5 \\
Due to infection & 2.5 \\
Due to congestive heart failure & 2.5 \\
\hline & \\
& \\
& \\
& \\
\hline
\end{tabular}

The newborn period was relatively normal in most cases. MUL was diagnosed in only one of the patients who had no affected siblings (table 1). Fourteen babies needed supplementary oxygen. Respiratory distress syndrome was diagnosed in three; two of them were born prematurely. Cardiac involvement was evident in only one baby with WollfParkinson-White syndrome causing paroxysmal supraventricular tachycardial attacks. One third of the newborns had serious feeding difficulties; 14 of them needed a nasogastric tube. Twelve babies were hospitalised from 14 to 55 days because of feeding difficulties or pulmonary problems.

\section{Later infancy}

The growth failure progressed over the first 2 years of life in 63 patients $(74 \%)$ with an SDS decrease of more than 1.0 (range -1.0 to -3.5 ). Catch up growth with a gain in length SDS of more than 1.0 (range 1.1 to 2.9 ) occurred in five $(6 \%)$ of the infants. One boy and two girls reached a length SDS above -2.0 at the age of 2.0 years; one girl grew steadily from birth staying at an SDS of -1.4. At the age of 2.0 years, length SDS was on average -4.4 (range 0.1 to -7.8 ) (fig 1). Weight gain was also poor. Mean relative weight for height at the age of 2.0 years was $-21 \%$ (range -35 to $3 \%$ ).

Half of the patients failed to thrive. Feeding difficulties were the most common clinical problem such that one third needed nasogastric feeding and five children needed a percutaneous gastrostomy (table 1). A high frequency of upper respiratory infections and middle ear infections was evident; pneumonias were diagnosed in nearly half of the infants by the age of 2.0 years (table 1). Episodes of respiratory failure induced by an infection occurred in $25 \%$ of the infants. Nine infants had to be resuscitated (table 1). 
Four and nine patients had congestive heart failure by the age of 1.0 and 2.0 years, respectively; four of them died.

Psychomotor development was normal or slightly delayed in a great majority of the infants. Mild hypotonicity was evident in nearly half of the infants, and a delay in both motor development and speech was noted in nearly one third. Age at the first steps and the first words ranged from 0.8 to 2.6 years (mean 1.2 years) and 0.9 to 3.1 years (mean 1.3 years), respectively. A mildly hydrocephalic skull with frontal bossing and abnormally wide fontanelles evoked suspicion of hydrocephalus in $15 \%$ of the infants (table 1).

\section{Findings at the time of diagnosis} Clinical findings

At the time of the diagnosis all but four (96\%) of the patients were below length SDS of -2.5. Nearly all the patients were gracile with relative macrocephaly, and thin extremities with proximal relative shortness and narrow shoulder (fig 2). The characteristic triangular face with high, broad forehead and low nasal bridge was present in over $90 \%$, and scaphocephaly with occipitofrontal bossing was seen in over $80 \%$ of the patients (table 2, fig 3)

Fifteen patients (18\%) had signs of heart disease (table 3 ). Ten of them met the criteria of congestive heart failure, and four had pericardial constriction necessitating pericardiectomy. One woman had been pericardiectomised at the age of 32 years ( 12 years before the diagnosis) due to constrictive pericarditis. Eighteen patients (15\%) had structural heart anomalies (table 3).

Thirty eight patients (45\%) had hepatomegaly and six of them had liver tumours. Wilms' tumour was detected in two patients before MUL was diagnosed. Both patients had nonsymptomatic haematuria with a palpable abdominal mass as the only signs. Renal and genital anomalies were observed in $18 \%$ and $9 \%$ of the patients, respectively (table 3 ).

A peculiar voice characterised by high pitch and slight coarseness was noted in nearly all of the prepubertal patients. Characteristic ocular findings ${ }^{16}$ were yellow dots in the midperiphery of ocular fundi, seen in $80 \%$ of the patients. Additional ocular findings were retinal hypopigmentation and pigment dispersion with clusters of pigment in the
Table 2 Dysmorphic features of appearance at the time of diagnosis in 85 patients with Mulibrey nanism

\begin{tabular}{ll}
\hline Feature & Frequency $(\%)$ \\
\hline Head & \\
Characteristic face: triangular face, high & 90 \\
and broad forehead; low nasal bridge & \\
and telecanthus & \\
Scaphocephaly & 81 \\
Hypoplastic tongue & 80 \\
Low set located posteriorly rotated ears & 54 \\
Dental crowding & 50 \\
High hairline & 45 \\
Wide fontanelles and sutures in infancy & 37 \\
Abnormally wide metopic suture & 31 \\
Cleft palate & 2 \\
Body & \\
Thin extremities & 99 \\
Accentuated lumbar lordosis & 96 \\
General gracility & 95 \\
Narrow shoulders & 94 \\
Small bell shaped thoracic cage & 94 \\
Barrell-like trunk & 92 \\
Cutaneus naevi flammei & 65 \\
Hypoplastic buttocks & 52 \\
Constitution & \\
Relative proximal shortness of limbs & 90 \\
Large head relative to stature & 81 \\
Large hands and feet relative to stature & 67 \\
Skeletal asymmetry & 15 \\
\hline & \\
&
\end{tabular}

retina. Two thirds of the patients had cutaneous naevi flammei, most commonly in the lower limbs $(80 \%)$.

\section{Radiological findings}

Slender long bones with thick cortex and narrow medullary channel (fig 4) and a low, shallow (J shaped) sella turcica (fig 5) were the characteristic radiological findings present in nearly all patients (table 4). More than half of the patients had true orbital hypertelorism. The orbital fossae were slightly upward slanting with laterally rotated axis (fig 5). Fibrous dysplasia was present in $15 \%$ of the patients, mostly in the lower limbs (fig 4). Six patients had fibrous dysplasia in more than two locations (range 2 to 4 ). A slight asymmetry
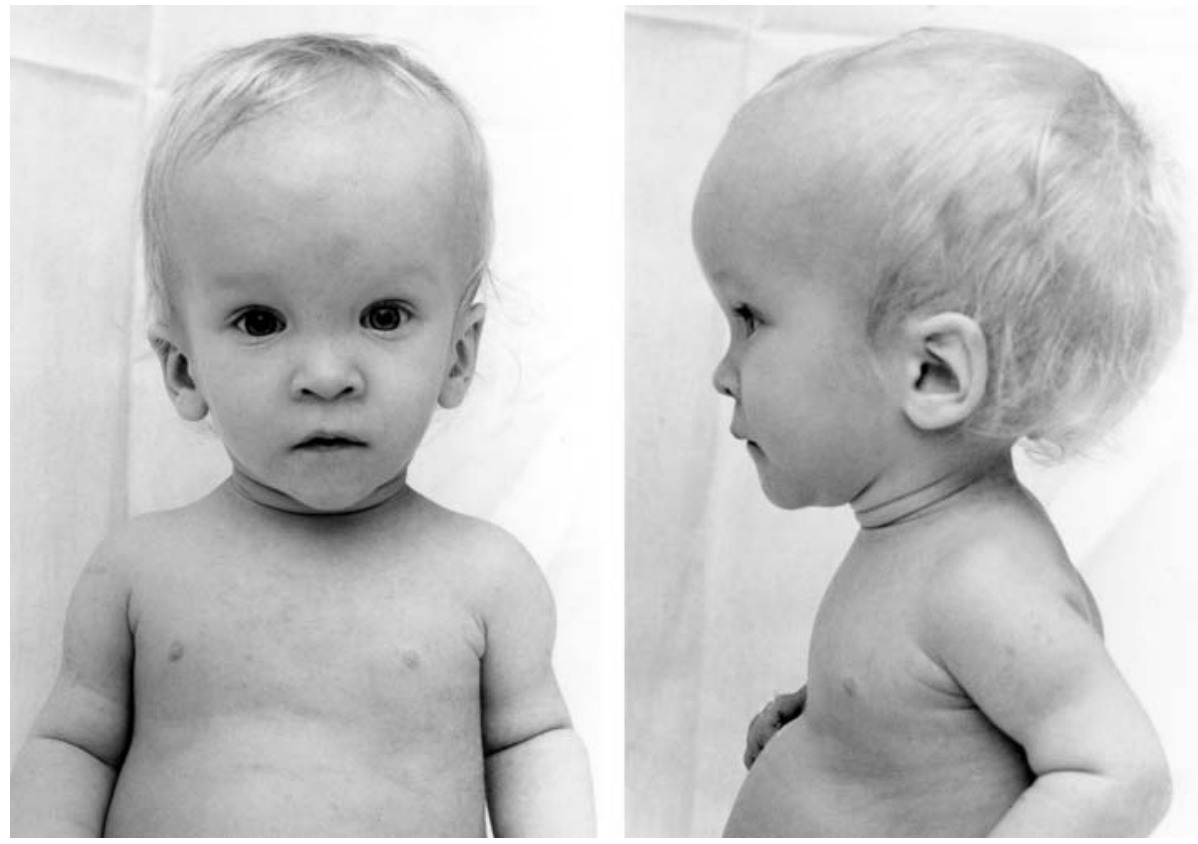

Figure 2 The characteristic craniofacial features; triangular face, low nasal bridge, high and broad forehead, and scaphocephaly with occipitofrontal bossing. 


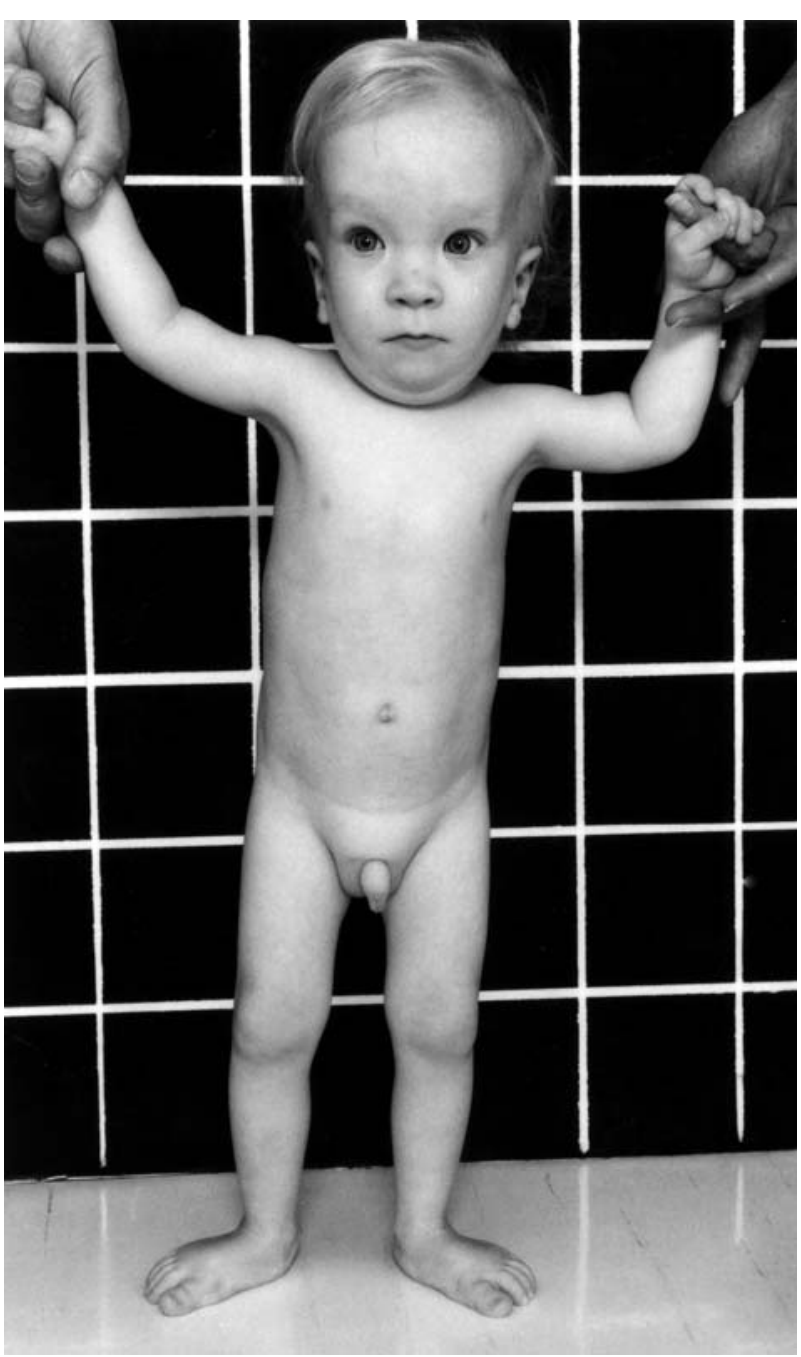

Figure 3 The characteristic appearance; triangular face, relative macrocephaly, general gracility, small bell shaped thoracic cage, and thin and proximally short limbs.

of extremities was noted in 17 patients $(20 \%)$, which in two thirds was due to shortening by fibrous dysplasia. The thoracic cage was small and bell shaped with unusually thin ribs in nearly all patients (table 4, fig 6). Twelve patients had an enlarged heart on chest radiography.

\section{Laboratory findings}

Serum levels of the aminotransferases were slightly elevated in nearly half of the patients, AST up to $110 \mathrm{U} / \mathrm{l}$ (mean 61 U/l) and ALT up to $193 \mathrm{U} / \mathrm{l}$ (mean $41 \mathrm{U} / \mathrm{l}$ ). Blood haemoglobin, white cells and platelets, glucose, gas analysis, and serum concentrations of electrolytes and creatinine were within the normal limits in the majority of patients. No other laboratory parameters were commonly abnormal.

\section{DISCUSSION}

In this work, we analysed retrospectively the clinical characteristics of patients with MUL in all 85 known Finnish patients at the time of diagnosis. At least 79 were homozygous for the Finn major mutation of the TRIM 37 gene. The results confirm that MUL is a distinct but variable entity. The most consistent findings were intrauterine onset growth failure and characteristic craniofacial features. Organ manifestations varied considerably in early childhood. In seven of the families, there was an affected sibling. In five of
Table 3 Organ manifestations at the time of diagnosis in 85 patients with Mulibrey nanism

\begin{tabular}{|c|c|c|c|c|}
\hline \multirow[b]{2}{*}{ Feature } & \multicolumn{3}{|c|}{ Findings in age groups } & \multirow[b]{2}{*}{$\begin{array}{l}\text { Frequency } \\
(\%)\end{array}$} \\
\hline & $\begin{array}{l}<1 \text { year } \\
(n=29)\end{array}$ & $\begin{array}{l}1-5 \\
\text { years } \\
(n=29)\end{array}$ & $\begin{array}{l}>5 \text { years } \\
(n=27)\end{array}$ & \\
\hline \multicolumn{5}{|l|}{ Heart } \\
\hline Signs of heart disease & 6 & 4 & 5 & 18 \\
\hline Cardiomegaly & 4 & 2 & 6 & 14 \\
\hline Dyspnoea & 4 & 1 & 5 & 12 \\
\hline $\begin{array}{l}\text { Prominent veins on } \\
\text { upper body }\end{array}$ & 3 & 1 & 5 & 11 \\
\hline Cardiac anomaly & 6 & 2 & 4 & 15 \\
\hline ASD & 3 & 1 & 2 & 8 \\
\hline VSD & 1 & 1 & 2 & 5 \\
\hline Open ductus arteriosus & 2 & 0 & 0 & 3 \\
\hline Heart failure (CHF) & 4 & 1 & 5 & 12 \\
\hline Pericardial constriction & 2 & 1 & 2 & 6 \\
\hline \multicolumn{5}{|l|}{ Liver } \\
\hline Hepatomegaly & 16 & 9 & 13 & 45 \\
\hline Hepatosplenomegaly & 4 & 1 & 4 & 11 \\
\hline Ascites & 3 & 0 & 6 & 11 \\
\hline Liver tumours & 2 & 2 & 2 & 9 \\
\hline \multicolumn{5}{|l|}{ Urogenital } \\
\hline Renal anomaly & 4 & 4 & 6 & 18 \\
\hline Hydronephrosis & 2 & 0 & 3 & 6 \\
\hline $\begin{array}{l}\text { Abnormally located } \\
\text { kidney }\end{array}$ & 1 & 1 & 2 & 5 \\
\hline Hypoplastic kidneys & 1 & 2 & 1 & 5 \\
\hline Horseshoe kidney & 1 & 1 & 0 & 2 \\
\hline Male genital anomaly* & & & & 6 \\
\hline Cryptorchidism & $1 / 13$ & $3 / 15$ & $1 / 11$ & 6 \\
\hline Female genital anomaly $\dagger$ & & & & 2 \\
\hline Hyperplastic labiae & $0 / 16$ & $1 / 14$ & $0 / 16$ & 1 \\
\hline Prominent clitoris & $0 / 16$ & $1 / 14$ & $1 / 16$ & 2 \\
\hline Wilms' tumour & 0 & 1 & 1 & 3 \\
\hline Benign renal neoplasia & 1 & 0 & 1 & 3 \\
\hline \multicolumn{5}{|l|}{ Nervous system } \\
\hline $\begin{array}{l}\text { Yellowish dots in } \\
\text { the ocular fundi }\end{array}$ & 20 & 17 & 24 & 79 \\
\hline Muscular hypotonicity & 23 & 19 & 15 & 68 \\
\hline $\begin{array}{l}\text { Large cerebral ventricles } \\
\text { and basal cisternas }\end{array}$ & 16 & 13 & 8 & 44 \\
\hline Slow speech development & 7 & 9 & 5 & 30 \\
\hline Slow motor development & 5 & 9 & 6 & 29 \\
\hline \multicolumn{5}{|l|}{ Skin } \\
\hline Cutaneous naevi flammei & 16 & 20 & 19 & 65 \\
\hline
\end{tabular}

these, the clinical findings were quite similar in the siblings, but in two families the phenotype showed great variation.

The clinical features have been previously reported. ${ }^{1} 2101617$ These reports, however, were based on small numbers of genetically unconfirmed patients. In our present series we verified the TRIM37 mutations and concentrated on the phenotype in infancy and at the time of diagnosis.

The clinical diagnosis of MUL is a challenge during the first months of life. Infants with intrauterine growth failure are a heterogenous group, and the facial features of patients with MUL are not unique in this group. In addition, relative macrocephaly with wide fontanelles might give a false impression of prematurity or hydrocephalus. However, the pre- and post-natal growth characteristics narrow the group in which MUL should be looked for. Generally, infants with MUL are both abnormally short and light for length. Additionally, they not only fail to catch up in postnatal growth, but their growth failure progresses. Of all newborns, $5 \%$ are born SGA, but only $1.5 \%$ are both short and light for length. ${ }^{18}$ Further, most $(>70 \%)$ healthy infants born SGA show a good catch up growth during the first few months of life. ${ }^{18}{ }^{19}$ Consequently, it is in the small subgroup of children born SGA and lacking postnatal catch up growth that the possibility of MUL should be considered. 


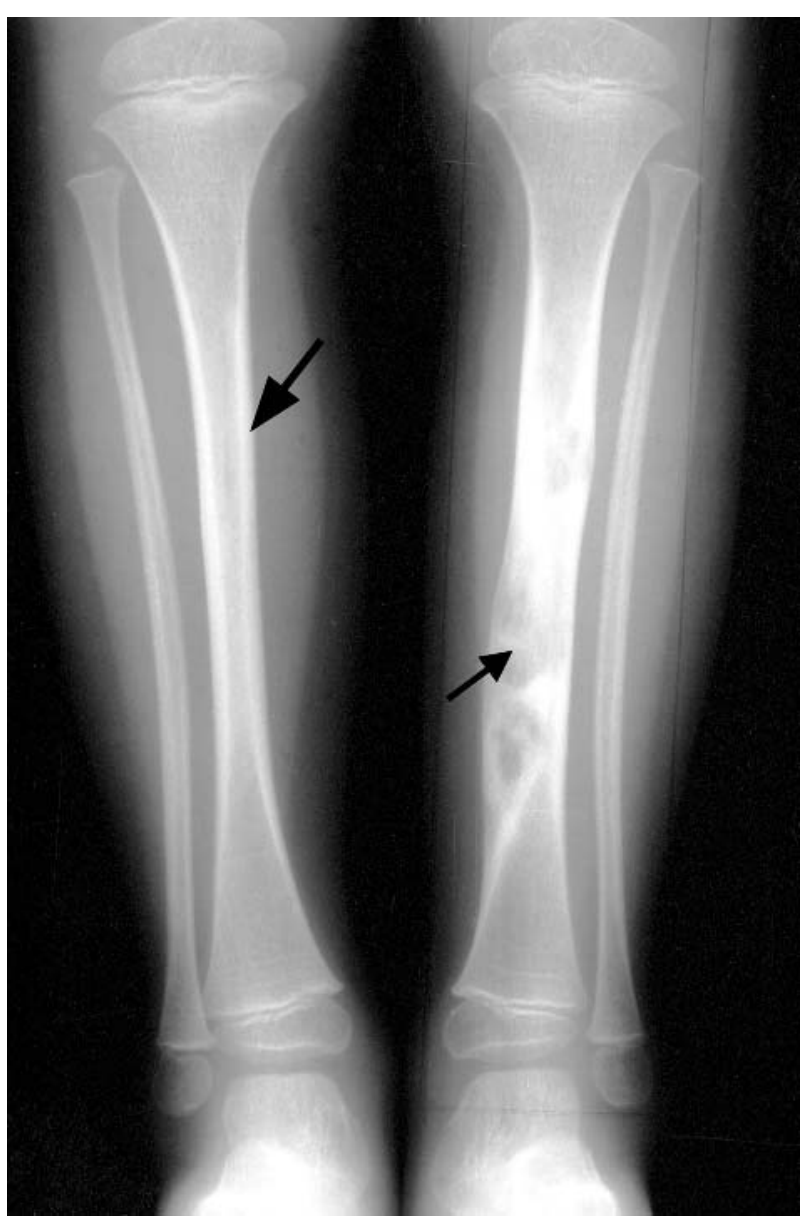

Figure 4 The characteristic $x$ ray findings: slender long bones with thick cortex and narrow medullary channel (big arrow). Fibrous dysplasia in the middle third of left tibia (small arrow).

Silver-Russell syndrome (SRS) is the other dysmorphic growth disorder characterised by severe intrauterine growth failure with absence of postnatal catch up growth. The fact that patients with MUL and SRS both are gracile with similar facial dysmorphism makes SRS the most important differential diagnosis. In early infancy, feeding difficulties occur in both MUL and SRS. These two conditions, however, develop their own distinct characteristics. Clinodactyly, small face with marked triangularity, micrognatia, downturned mouth corners, and skeletal asymmetry with hemihypertrophy are
Table 4 Radiological findings in 85 patients with Mulibrey nanism

\begin{tabular}{ll}
\hline Feature & Frequency $(\%)$ \\
\hline Head & 90 \\
Occiptofrontal bossing & 89 \\
Low and shallow (J shaped) sella turcica & 80 \\
Laterally rotated orbital fossae & 71 \\
Small hypoplastic face & 64 \\
Orbital hypertelorism & 43 \\
Large cerebral ventricles and cisternas & \\
Body & 96 \\
Accentuated lumbal lordosis & 94 \\
Bell shaped thorax with unusually thin ribs & 93 \\
Extemities & 25 \\
Slender long bones & \\
Fibrous dysplasia of long bone & \\
\hline
\end{tabular}

characteristics of SRS. ${ }^{20-22}$ Hepatomegaly, heart failure due to pericardial constriction or cardiomyopathy, yellow dots in ocular fundi, cortical thickening, and fibrous dysplasia of long bone do not occur in SRS. SRS, however, is considerably variable both clinically ${ }^{21}$ and genetically. ${ }^{23}$ A mild phenotype, for example, is observed in SRS patients with maternal uniparenteral disomy of chromosome $7 .^{22}$ Furthermore, atypically mild cases with MUL have been diagnosed in three Finnish infants since the analysis of TRIM37 became available.

The TRIM37 protein is localised to peroxisomes, but its function is unknown. It is expressed in several tissues. ${ }^{14}$ Thus, it is no surprise that MUL shares features with other peroxisomal disorders. These include growth failure, facial dysmorphism with midface hypoplasia, retinal pigmentary changes, skeletal dysplasia, skin changes, cardiac involvement, muscular hypotonicity, and hepatomegaly. In particular, Refsum's disease, with its cardiac manifestations such as cardiomyopathy leading to congestive heart failure, resembles many of the features of MUL. $^{24}$ Lack of major neurological manifestations and mental retardation are the most notable differences between Mulibrey nanism and the known peroxisomal disorders. The only neurological dysfunctions occurring in MUL are mild muscular hypotonicity and slight delay in motor and speech development.

Constrictive pericarditis ${ }^{25}$ with restrictive cardiomyopathy, when present, dominates the clinical state as well as the prognosis. ${ }^{26}$ However, only $12 \%$ of our patients had congestive heart failure at the time of diagnosis (median 2.1 years) and even half of the adult patients were free of major heart problem. ${ }^{26}$ In contrast, almost all patients
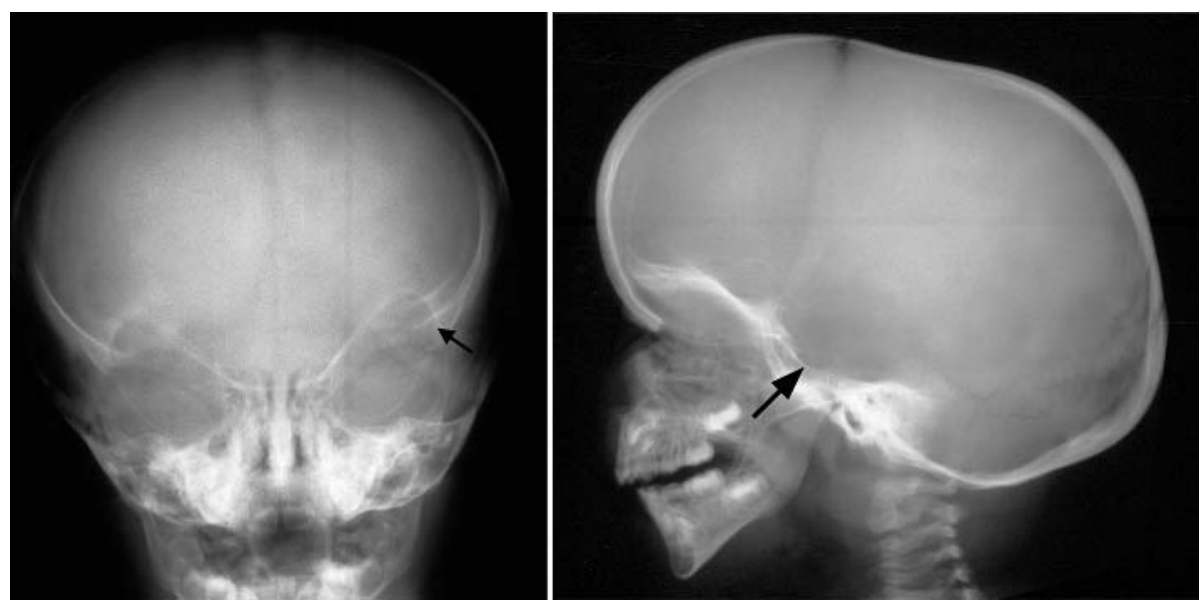

Figure 5 A characteristic cranium with high and broad forehead, small face, orbital hypertelorism, and scaphocephaly with occipitofrontal bossing and J shaped sella turcica (large arrow). Note the characteristic slightly upward slanting orbital fossae with the laterally rotated axis (small arrow). 


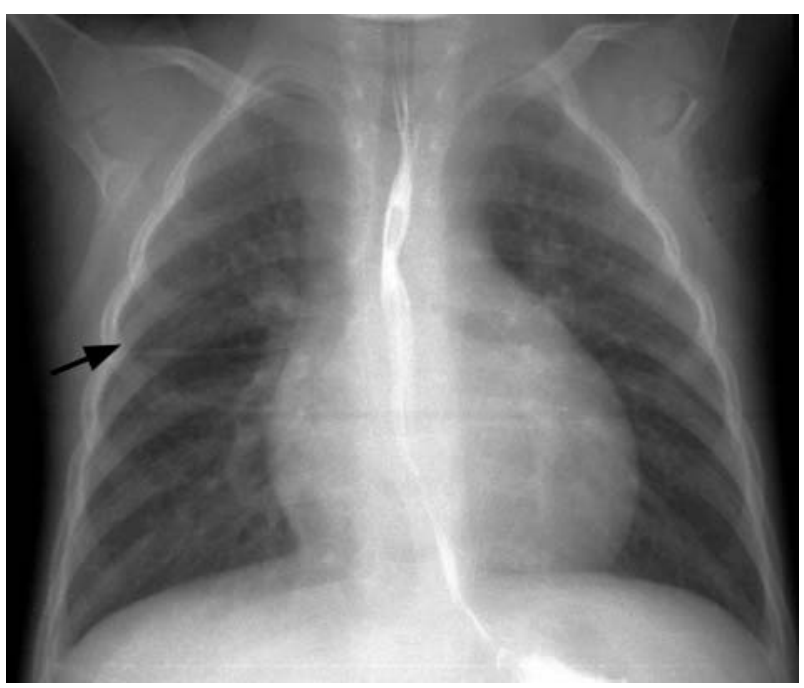

Figure 6 The small bell shaped thoracic cage with thin ribs (arrow). Note the characteristically rounded heart shadow frequently seen in children with MUL.

reported from outside Finland were notably compromised by the heart disease. Presumably, while MUL is relatively well known to Finnish paediatricians, it is elsewhere mostly recognised by its characteristic heart disease. As the heart involvement is critical for the prognosis, its early diagnosis is of major importance.

Hepatomegaly was obvious in half of the patients at the time of the diagnosis. Histopathological studies showed chronic passive congestion, indicating that the liver enlargement is an early clinical sign of the heart disease. At the time of the diagnosis, $10 \%$ of the patients had liver tumours as detected by radiological examinations or autopsy. The tumours differed in size and were often multifocal. Histopathological analysis of the tumour samples revealed hamartomas with fibrosis and lipid degenaration. To reveal these lesions, abdominal ultrasonography is regularly performed, and if suspicion of liver tumours arise, MRI is indicated. Beside liver tumours, two of the patients had Wilms' tumour as published previously for one Finnish and one non-Finnish patient. ${ }^{611}$ The biological basis of the tumour development remains to be solved.

Feeding difficulties are a major clinical problem during infancy. In general, swallowing is poorly coordinated in infants born prematurely or SGA and the degree of dysfunction is inversely related to the degree of immaturity and growth failure. ${ }^{27} 28$ The oral anatomy of mature infants are particularly well suited for eating by sucking, with the tongue being relatively large, filling the oral cavity. ${ }^{27} 29$ Infants with MUL, however, have a hypoplastic and anteriorly triangular shaped tongue. This is evident in nearly $70 \%$ of these infants. The craniofacial hypoplasia, maturational delay, ${ }^{17}$ and muscular hypotonicity all probably contribute to the feeding difficulty. Early recognition and management are important because dysphagia poses a threat to the child's respiratory function and nutrition, and may also contribute to the failure of catch up growth.

Patients with MUL are prone for respiratory tract infections and have an exceptionally high risk for pneumonia. No immunological defects have been demonstrated in MUL and the basis of this susceptibility is not known. Possibly, the pneumonia may result from aspiration. Interestingly, infections quite easily lead to respiratory failure, suggesting that the ability to cope with the stress of an infection is subnormal. In our study, two patients
Table 5 Diagnostic signs and their prevalence in 85 patients with Mulibrey nanism (46 females and 39 males) For the diagnosis, three major signs with one minor sign are required, or two major signs with three minor signs

\begin{tabular}{ll}
\hline Signs & Frequency $(\%)$ \\
\hline Major signs & \\
Growth failure (A or B or C) & \\
A) small for gestational age (SGA) & 95 \\
lacking catch up growth & 94 \\
B) height in children 2.5 SDS below & \\
population mean for age & 90 \\
$\quad$ C) height in adults 3.0 SDS below population & \\
mean & \\
Characteristic radiological findings (A or B) & 93 \\
A) slender long bones with thick cortex & \\
and narrow medullar channels & \\
B) low and shallow (J-shaped) sella turcica & 89 \\
Characteristic craniofacial features & 90 \\
Scaphocephaly, triangular face, high and \\
broad forehead, low nasal bridge and telecanthus \\
Characteristic ocular findings \\
Yellowish dots in retinal mid peripheral region \\
Mulibrey nanism in a sibling & 79 \\
Minor signs & 17 \\
Peculiar high pitched voice & 96 \\
Hepatomegaly & 70 \\
Cutaneous naevi flammei & 65 \\
Fibrous dysplasia of long bone & 25 \\
\hline & \\
\hline
\end{tabular}

died in infancy from an infection. At autopsy, both were found to have adrenal cortical hypoplasia. Subnormal stress tolerance could be due to adrenal hypoplasia with adenocortical failure.

While growth failure, the craniofacial features, and hepatomegaly are important clinical consequences of MUL, they are also common features of other dysmorphic conditions. Hence, the characteristic ocular and radiological findings, cutaneous naevi flammei, fibrous dysplasia of the long bones, or signs of the Mulibrey heart disease are confirmatory of the MUL diagnosis. Based on our present findings and clinical experience, we propose revised diagnostic criteria for Mulibrey nanism (table 5). Importantly, while none of the clinical features was constant, 99\% of our patients presented with at least three of the major and one of the minor signs. One patient had only two major signs, but he had three of the minor signs (table 5).

In conclusion, we have delineated the phenotype and defined the clinical diagnostic criteria in the genotypically homogenous series of all known Finnish patients with Mulibrey nanism. Different TRIM37 mutations may not lead to exactly the same phenotype. However, it is important that infants born SGA, lacking postnatal catch up growth, and having poor weight gain, hepatomegaly, and characteristic craniofacial features should evoke suspicion of Mulibrey nanism. Although rare, this condition might also underlie congestive heart failure and failure to thrive in absence of a clear characteristic dysmorphology.

\section{ACKNOWLEDGEMENTS}

We want to thank the families of our patients. We also express our gratitude to the patients' local physicians, particularly Drs S Friman, J Komulainen, H-L Lenko and M Pere, for decades of co-work. This study was supported by grants from the Finnish Foundation for Paediatric Research, the Finnish Academy, and Finska Läkaresällskapet.

\section{Authors' affiliations}

N Karlberg, H Jalanko, J Perheentupa, M Lipsanen-Nyman, The Hospital for Children and Adolescents, Biomedicum Helsinki, University of Helsinki, 00029 HYKS, Finland 


\section{REFERENCES}

1 Perheentupa J, Autio S, Leisti S, Tuuteri L, Raitta C. Mulibrey nanism: dwarfism with muscle, liver, brain and eye involvement. Acta Pediatr Scand 1970;59(Suppl 206):74-5

2 Perheentupa J, Autio S, Leisti S, Raitta C, Tuuteri L. Mulibrey nanism, an autosomal recessive syndrome with pericardial constriction. Lancet 1973;2:351-55.

3 Thoren C. So-called Mulibrey nanism with pericardial constriction. Lancet 1973;29:731

4 Cumming GR, Kerr D, Ferguson CC. Constrictive pericarditis with dwarfism in two siblings (mulibrey nanism). J Pediatr 1976;88:569-72.

5 Voorhess ML, Husson GS, Blackman MS. Growth failure with pericardial constriction. The syndrome mulibrey nanism. Am J Dis Child 1976;130:1146-8.

6 Similä $S$, Timonen $M$, Heikkinen $E$. A case of mulibrey nanism with associated Wilms' tumor. Clin Genet 1980;17:29-30.

7 Sanchez-Corona J, Moran-Vazquez O, Rivera H, Hernandes A, SerranoLucas JI, Ocampo-Campos R, Lasso-Avalos L, Maria-Cantus J. Mulibrey nanism in a Mexican child. Bol Med Hosp Infant Mex 1983:40:45-8.

8 Cotton JB, Rebelle C, Boiso A, Ladreyt-Ponchon JP, Maillet J. Familial intrauterine nanism with constrictive pericarditis, the MuLiBrEy syndrome. Pediatrie 1988;43:197-203.

9 Haraldsson A, van der Burgt CJ, Weemaes CM, Otten B, Bakkeren JA, Stoelinga GB. Antibody deficiency and isolated growth hormone deficiency in a girl with Mulibrey nanism. Eur J Pediatr 1993;152:509-12.

10 Lapunzina P, Rodriguez JI, de Matteo E, Gracia R, Moreno F. Mulibrey nanism: three additional patients and a review of 39 patients. Am J Genet 1995; 55:349-55

11 Seemanova E, Bartsch O. Mulibrey nanism and Wilms' tumor. Am J Med Genet 1999;85:76-8.

12 Jagiello P, Hammans C, Wieczorek S, Arning L, Stefanski A, Strehl H, Epplen JT, Gencik M. A novel splice site mutation in the TRIM37 gene causes Mulibrey nanism in a Turkish family with phenotypic heterogeneity. Hum Mut 2003;21:630-35.

13 Avela K, Lipsanen-Nyman M, Idänheimo N, Seemanova E, Rosengren S, Mäkelä TP, Perheentupa J, de la Chapelle A, Lehesjoki AE. Gene encoding a new RING-B-box-Coiled-coil protein is mutated in mulibrey nanism. Nat Genet 2000;25:298-301.

14 Lehesjoki AE, Reed VA, Mark Gardiner R, Greene ND. Expression of MUL, a gene encoding a novel RBCC family ring-finger protein, in human and mouse embryogenesis. Mech Dev 2001;108:221-5.

\section{CORRECTION}

doi: 10.1136/jmg.2003.010363corrl

DFNA49, a novel locus for autosomal dominant nonsyndromic hearing loss, maps proximal to DFNA7/DFNM1 region on chromosome 1q21-q23 (J Med Genet 2003;40:832-836).

Owing to a fault in the production process an error was introduced to figure 2 of this paper. The last gene at the bottom of the figure should correspond to CASQ1, and not to ATP1A2, which is mapped just above it. We apologise for this error.
15 Kallijärvi J, Avela K, Lipsanen-Nyman M, Ulmanen I, Lehesjoki AE. The TRIM37 gene encodes a peroxisomal RING-B-box-Coiled-coil protein: Classification of mulibrey nanism as a new peroxisomal disorder. Am J Hum Genet 2002;70:1215-28.

16 Raitta C, Perheentupa J. Mulibrey nanism; an inherited dysmorphic syndrome with characteristic ocular findings. Acta Ophtalmol Suppl 1974;123:162-71.

17 Myllärniemi S, Koski K, Perheentupa J. Craniofacial and dental study of mulibrey nanism. Cleft Palate J 1978;15:369-77.

18 Albertsson-Wikland K, Karlberg J. Natural growth in children born small for gestational age with and without catch-up growth. Acta Pediatr 1994;83(Suppl 399):64-70.

19 Hokken-Koelega ACS, De Ridder MAJ, Lemmen RJ, Den Hartog H, De Muinck Keizer-Schrama SMPF, Drop SLS. Children born small for gestational age: Do they catch up? Pediatr Res 1995;38:267-71.

20 Escobar V, Gleiser S, Weaver DD. Phenotypic and genetic analysis of the Silver-Russell syndrome. Clin Genet 1978;13:278-88.

21 Price SM, Stanhope R, Garrett C, Preece MA, Trembath RC. The spectrum of Silver-Russell syndrome: a clinical and molecular genetic study and new diagnostic criteria. J Med Genet 1999;36:837-42.

22 Hannula K, Kere J, Pirinen S, Holmberg C, Lipsanen-Nyman M. Do patients with maternal uniparenteral disomy for chromosome 7 have a distinct mild Silver-Russell phenotype? J Med Genet 2001;38:273-8.

23 Hitchins MP, Stainer P, Preece MA, Moore GE. Silver-Russell syndrome: a dissection of the genetic aetiology and candidate chromosomal regions. J Med Genet 2001;38:810-19.

24 Leys D, Petit H, Bonte-Adnet C, Millaire A, Fourrier F, Dubois F, Rosseaux M, Ducloux G. Refsum's disease revealed by cardiac disorders. Lancet 1989;1:621.

25 Tuuteri L, Perheentupa J, Rapola J. The cardiopathy of mulibrey nanism, a new inherited syndrome. Chest 1974;65:628-31.

26 Lipsanen-Nyman M, Perheentupa J, Rapola J, Sovijärvi A, Kupari M. Mulibrey heart disease: Clinical manifestations, long-term course and results of pericardiectomy in a series of 49 patients born before 1985. Circulation 2003;107:2810-15.

27 Tuchman DN. Dysfunctional swallowing in the pediatric patient: clinical considerations. Dysphagia 1988;2:203-8.

28 Selley WG, Ellis RE, Brooks WA. Coordination of sucking, swallowing and breathing in the newborn: its relationship to infant feeding and normal development. Br J Disord Commun 1990;25:311-27.

29 Stevenson RD, Allaire JH. The development of normal feeding and swallowing. Pediatr Clin North Am 1991;38:1439-53. 